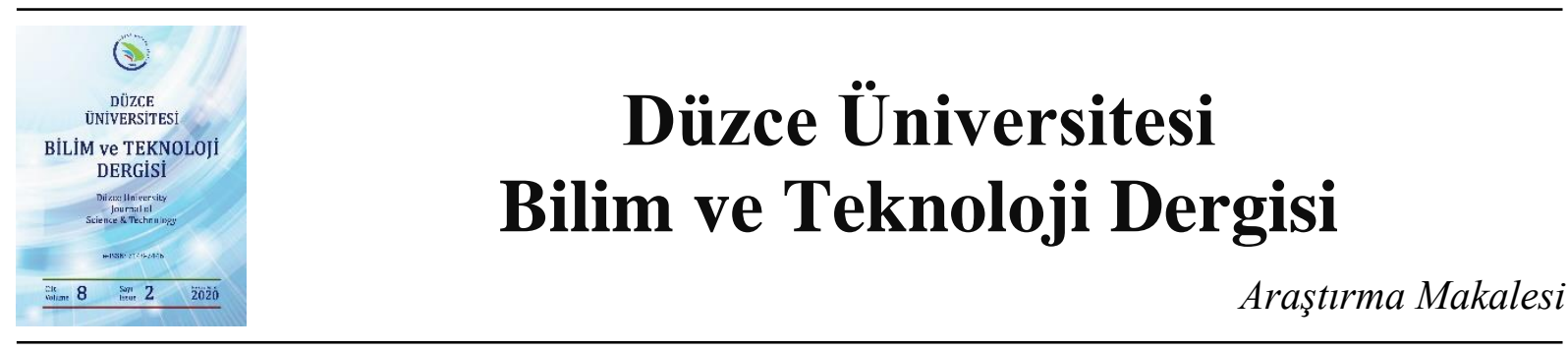

\section{Güç Sistemlerinde Geçici Kararlılık için UPFC, GSSC ve Alt-Geçici Dinamik Modellerin Geliştirilmesi}

\author{
Kenan DÖŞOĞLU ${ }^{\text {a,* }}$ \\ ${ }^{a}$ Elektrik-Elektronik Mühendisliği Bölümü, Teknoloji Fakültesi, Düzce Üniversitesi, Düzce, TÜRKİYE \\ * Sorumlu yazarın e-posta adresi: kenandosoglu@duzce.edu.tr
}

DOI: $10.29130 /$ dubited.688782

\begin{abstract}
ÖZET
Güç sistemlerinin çalışması esnasında bazı nedenlerden dolayı kararsızlık problemleri ile karşı karşıya kalmaktadır. Kararsızlık problemlerinin en önemli nedenlerinden birisi kısa devre durumlarıdır. Kısa devre güç sistemlerinde salınımlara sebep olmaktadır. Bu salınımları ortadan kaldırmak için Güç Salınım Sönümleme Kontrolü (GSSC) kullanılmaktadır. Bu çalışmada, güç sistemlerinde osilasyonların sönümlenmesi için Kundur 4 makinalı sistemde farklı senaryolar ile analizler gerçekleştirilmiş̧tir. Ayrıca çok makinalı sistemde kullanılan senkron generatörlerin alt-geçici durum dinamik modellemeleri bu çalıșmada tercih edilmiștir. Birleștirilmiş Güç Akışı Kontrolü (UPFC), GSSC ve alt-geçici durum dinamik modellemelerinin kullanıldığı ve kullanılmadığı durumlarda karşılaştırmalar yapılmıştır. Elde edilen sonuçlar ile geliştirilen modellerin geleneksel olarak kullanılan modellere göre sistemi kısa sürede kararlı hale getirdiği ve salınımların azalttı̆̆g görülmüştür.
\end{abstract}

Anahtar Kelimeler: UPFC, GSSC, Senkron generatör, Alt-geçici durum dinamik modelleri

\section{Enhancement of UPFC, PODC and Sub-Transient Dynamic Model for Transient Stability in Power Systems}

\begin{abstract}
During power system operation, it faces instability problems thanks to some reason. One of the most important causes of instability problems is short circuit conditions. It causes oscillations in power systems. Power Swing Damping Control (PSDC) is used to eliminate these oscillations. In this study, analyzes were carried out with different scenarios in Kundur 4 machine system in order to dampen oscillations in power systems. In addition, sub-transient state dynamic models of synchronous generators used in multi-machine system are preferred in this study. Comparisons were made when Unified Power Flow Controller (UPFC), PSDC, and sub-transient state dynamic models were used and not used. It has been observed that the models developed with the results obtained have stabilized the system in a short time and the oscillations decreased compared to the traditionally used models.
\end{abstract}

Keywords: UPFC, PSDC, Synchronous generators, Sub-transient state dynamic models 


\section{GIRIS}

Çok baralı güç sistemlerinde, değişen çalışma koşullarıyla kararlı bir alanda çalışması zorlu bir mesele olarak görülmektedir. Güç sistemlerinin çalışmasında güvenilirlik ve bozucu etki sonucunda istenilen sistem dinamik performansının korunması için istenilen şartlar bulunmaktadır. Sistem kararlılı̆̆ ve güvenilirliği ile birlikte yüksek düzeyde güç aktarımı sağlayan yeni kavramlar ve teknolojiler son zamanlardaki ilgi alanlarındandır. Yüksek güçlü yarı iletken tabanlı cihazlar ve kontrol teknolojileri alanında yapılan ilerlemeler sayesinde çok baralı sistemlerde güvenirlik ve çalışma koşullarının iyileştirilmesi sağlanmıştır. Ancak çok baralı güç sistemlerinde, sönümleme olaylarında azalmalar ve elektromekanik salınımların ortaya çıkmasına sebep olmaktadır. Güç elektroniği tabanlı olarak geliştirilen GSSC tabanlı Esnek AC Transmission System (FACTS) cihazları ile bu kararsızlık durumları minimum düzeye indirilmektedir. FACTS cihazları içerisinde en gelişmiş yapıda olan eleman ise UPFC'dir. GSSC tabanlı UPFC güç sisteminde salınımların sönümlenmesinde önemli bir avantaja sahiptir.

UPFC'nin güç salınım sönümlenmesi için kontrol parametrelerinin elde edilmesi hankel, minimum ve direk moment tekil nokta analizi ile gerçekleştirilmiştir. Şebekede durum değişmelerinin anlık olarak takibi sağlanarak arıza esnasında oluşacak olan salınımlar geliştirilen modeller sayesinde azaltılmıştır [1]. Güç salınımların sönümlenmesi için kullanılan UPFC'de merkezi koordinat kontrol modeli geliştirilmiştir. Çok makinalı sistemde kullanılan koordinat kontrol modeli ilave sönümleme modelinde farklı PSS modelleri ile karşılaştırılarak en uygun olan çalışma metodu belirlenmiştir [2]. Çok makinalı sistemde UPFC'de geliştirilen diğer bir çalışma ise modifiye edilmiş olan Phllips-Heffron Modeldir. Geliştirilen bu model ile UPFC için sistem değişimlerindeki en optimum parametrelerin elde edilmesinin yanısıra sistemdeki özdeğer analiz durumları detaylı olarak incelenmiştir [3]. UPFC'nin düşük frekans çalışmaları için sistemde değişik çalışma kontrol sinyallerinin belirlenmesi ve optimum değerlerin gözlemlenmesi için adaptif güç salınım sönümleme modeli kullanılmıştır. Benzetim çalışmasında zaman model adaptasyonu ile geçici kararlılık durumlarında geliştirilen adaptif güç salınım sönümleme ile etkili sonuçlar elde edilmiştir [4]. İletim hatlarında güç akışı esnasında sistemde oluşan bozucu etkileri sonucunda meydana gelen güç salınımlarını kısa süre içerisinde ortadan kaldırmak için UPFC modeli geliştirilmiştir. Belirli referans değerinde oluşabilecek salınım durumlarına karşı UPFC'nin sistem dinamiği açısından iyi sonuçlar verdiğim görülmüş̧ür [5]. Farklı bir çalışmada farklı denetleyicilerin UPFC üzerindeki etkileri güç salınım sönümlenmesi açısından incelenmiştir. Sistemde oluşan bozucu etki durumlarına karşı UPFC'de oransal integral türev denetleyicisi, oransal integral denetleyicisi ve Ziegler-Nichols denetleyicileri arasında karşılaştırmalar yapılarak en uygun model için değerlendirmeler yapılmıştır [6-8]. Tek makinalı ve çok makinalı sistemde geçici durum analizi için salınım durumları eş zamanlı koordinasyon kontrolü için UPFC, GSSC ve PSS modelleri kullanılmıştır. Sistemin dinamik kararlılık analizinde düşük frekans salınımları için UPFC, GSSC ve PSS modellerinin genel olarak karşılaştırmaları ve yorumlamaları yapılmıştır [9]. Güç sistemlerinde arıza analizinde oluşan salınımların sönümlenmesi için UPFC'de decomposition tabanlı GSSC geliştirilmiştir. Denetleyiciler için kullanılan kontrol parametrelerinin doğrusal hale getirilmesi ile oluşturulan GSSC modelde çok makinalı sistemde dinamik zaman cevapları incelenmiş olup, sistem üzerindeki etkiler analiz edilmiştir [10].

Genel olarak literatürde UPFC, GSSC ve PSS modellerinin birlikte yaygın olarak kullanıldığ görülmektedir. Yapılan bu çalışmada literatürden farklı olarak UPFC'nin GSSC ve alt-geçici durum dinamik derece modellerinin kullanılması ile farklı bir analiz yöntemi belirlenmiştir. Farklı analiz yönteminin kullanılması ile karşılaştırmalar ve yorumlar yapılmıştır. UPFC, GSSC ve alt-geçici durum dinamik derece modellerinin kullanılması ile benzetim çalışmasında etkili sonuçlar elde edilmiştir. 


\section{UPFC MODELI}

Şekil 1'de yer alan devre modeli incelendiğinde, UPFC'nin şönt transformatör, seri transformatör, VSC1, VSC2 ve kontrol ünitesinden oluştuğu görülmektedir.

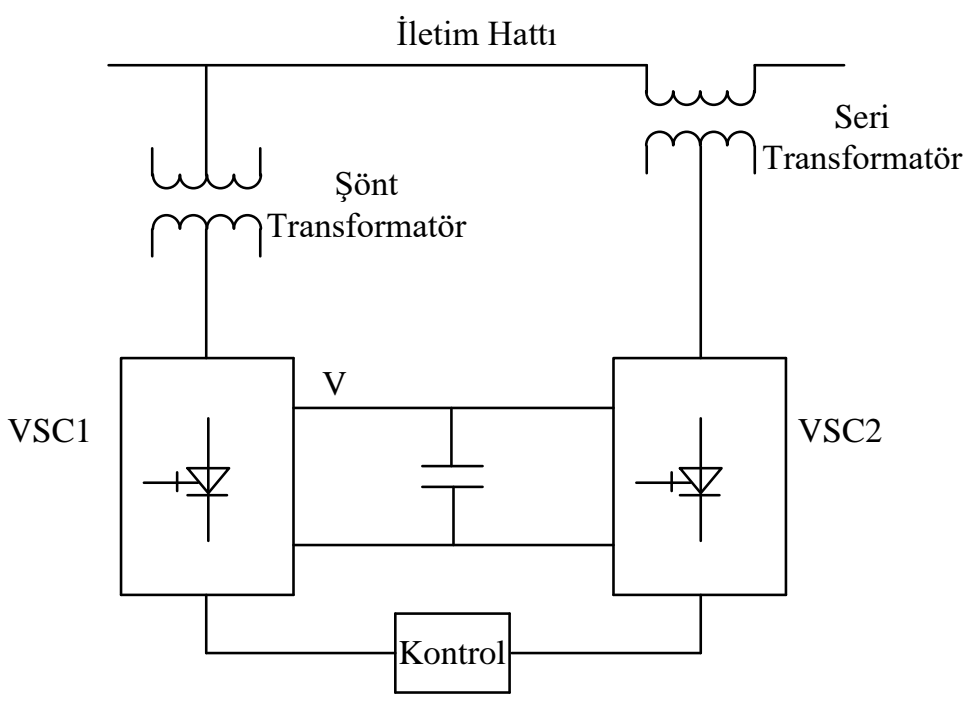

Şekil 1. UPFC devre modeli

UPFC devre modelinde, kullanılan gerilim kaynaklı eviricilerden oluşan tristör tetikleme devreleri yer almaktadır. Bu eviriciler VSC1 ve VSC2 olarak etiketlendirilmiş olup, çalıştırılması bir DC depolama kapasitörünün sağladığı ortak bir DC bağlantı ile gerçekleştirilir. $\mathrm{Bu}$ düzenleme ile $\mathrm{AC}$ güç dönüştürücüde aktif güç, iki eviricinin $\mathrm{AC}$ terminalleri ile her bir evirici arasındaki iki yönde serbest bir şekilde akabilir ve AC güç dönüştürücü, kendi AC çıkış terminalinde reaktif gücü çeker ve aynı zamanda bağımsız olarak üretebilir. UPFC'nin eviriciler arasında güç enjekte etme modelinde, iki bağlantı transformatöründeki reaktans bağlı empedans ve iki evirici devresinin çıkış gerilimi dalga boyu temel elemanlarının sürekli durum çalışmasındaki durumlarına göre oluşturulmaktadır. Bu model UPFC'nin sürekli durum analizinde etkisini anlayabilmemizi sağlamakta ve dahası sürekli durum yük akışı ile birlikte çalışma olanaklarını sunmaktadır [11]. UPFC güç enjekte modelinde, sistemin temel fonksiyonunda seri gerilim kaynaklı evirici bulunmadığı için, sürekli durum çalışmada yaklaşık bir eşdeğer model oluşturulmaktadır. Şekil 2'de gerilim kaynaklı eviricinin seri bağlantısı verilmiştir.

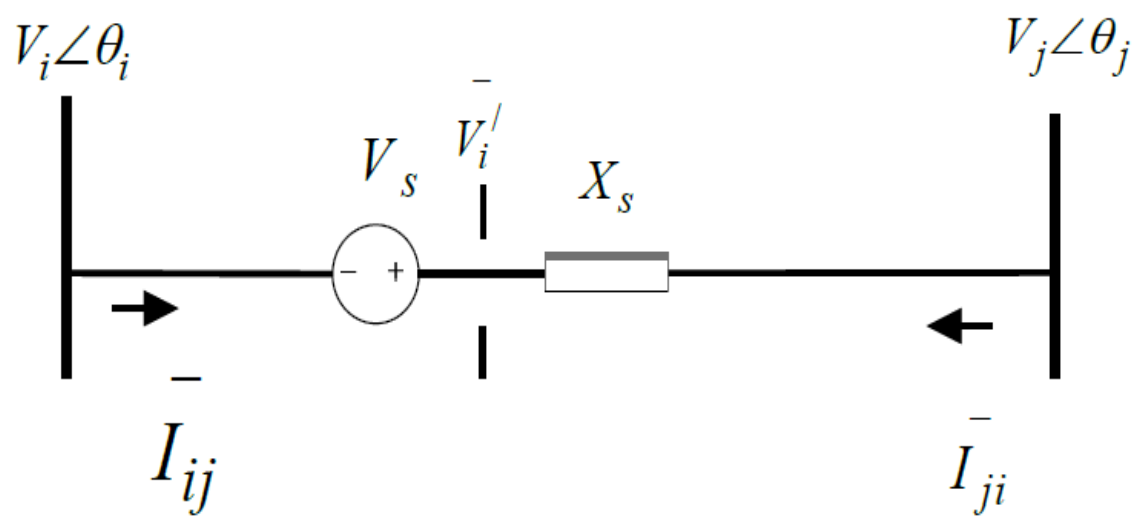

Şekil 2. UPFC'nin iki makinalı sistemde gösterimi

Şekil 2'de i ve j baraları kullanılmaktadır ve referans vektör olarak i barasının gerilimi alınmaktadır. Bu gerilim ifadesi denklem (1)'de gösterilmiştir. 
$\stackrel{-\prime}{V_{i}^{\prime}}=V_{s}+V_{i}$

Burada, $V_{i}$ i barasının referans vektörünü, Vs kaynak gerilimini ve Vi i barasının gerilimini ifade eder. Vs kaynak geriliminin gösterimi denklem (2)'de verilmiştir.

$V_{s}=r V_{i} e^{j \gamma}$

Bu denklemde, özel değerler olarak adlandırılan $\mathrm{r}$ ve $\Upsilon$ ifadelerinden $\mathrm{r} 0 \leq r \leq r_{\text {maksimum }}$ aralığında ve $\Upsilon 0 \leq \gamma \leq 2 \pi$ aralığında değerler almaktadır. Sürekli durum UPFC matematiksel modelinde iletim hattına paralel bağlı akım kaynağı modeli kaynak gerilimi ile yer değiştirmiştir. Elde edilen yeni devre modeli Şekil 3'te yer almaktadır.

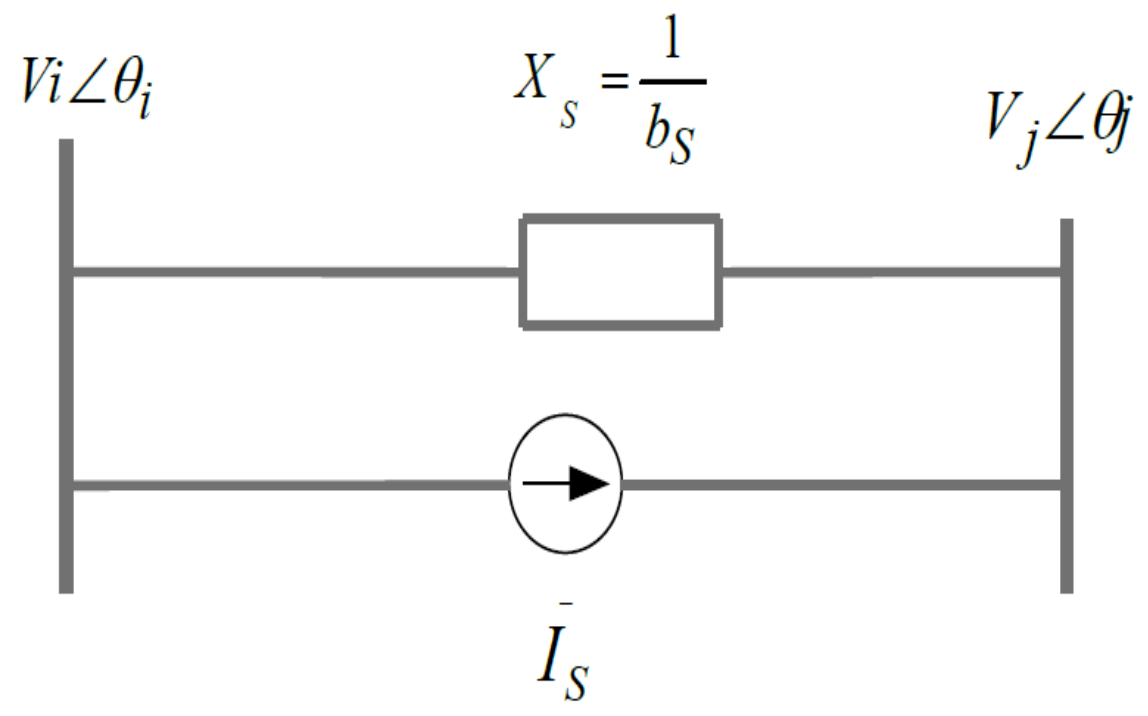

Şekil 3. Akım kaynağı ile elde edilen yeni devre modeli

Yeni modelde kullanılan akım kaynağı ve kaynak reaktansı ifadeleri denklem (3) ve denklem (4)'te gösterilmiştir.

$I_{s}=-j b_{s} V_{s}$

$b_{s}=\frac{1}{X_{s}}$

Burada, $\mathrm{I}_{\mathrm{s}}$ akım kaynağından geçen akımı, $\mathrm{b}_{\mathrm{s}}$ kaynak süseptansını ve $\mathrm{X}_{\mathrm{s}}$ kaynak reaktansını ifade eder. Baralar arasındaki görünür güç ifadelerine Denklem (5) ve Denklem (6)'da yer almaktadır.

$S_{i s}=-V_{i}\left(-I_{s}\right)^{*}$

$S_{j s}=-V_{j}\left(I_{s}\right)^{*}$

Yukarıda verilen eşitliklerin birbiri yerine yazılarak düzenlenmesiyle, yeni elde edilen ifade denklem (7)'de gösterilmiştir.

$S_{i s}=V_{i}\left[j b_{s} r V_{i} e^{j \gamma}\right]$ 
Euler teoreminin kullanılması ile oluşan yeni eşitlikler Denklem (8) ve Denklem (9)'da verilmiştir.

$$
\begin{aligned}
& S_{i s}=V_{i}\left[e^{j(\gamma+90)} b_{s} r V_{i}\right] \\
& S_{i s}=V_{i}^{2} b_{s} r[\cos (-\gamma-90)+j \sin (-\gamma-0)]
\end{aligned}
$$

Denklem (9)'un trigonometrik eşitliklerin kullanılarak sadeleştirilmiş hali Denklem (10)'da gösterilmiştir.

$S_{i s}=b_{s} r V_{i}^{2} \sin \gamma-j b_{s} V_{i}^{2} \cos \gamma$

Benzer eşleştirmenin Denklem (6)'da yapılması ile elde edilen yeni eşitlik ise Denklem (11)'de gösterilmiştir.

$S_{j s}=V_{j}\left[-j b_{s} r V_{J} E^{J \gamma}\right]$

UPFC'de aktif güç şönt evirici devresi tarafından seri bağlı olan evirici devresi aracılığıyla sağlanmaktadır. Kayıpların ihmal edildiği durumda evirici devrelerindeki aktif güç eşitlikleri Denklem (12)'de gösterilmiştir.

$P_{\text {evirici } 1}=P_{\text {evieici } 2}$

Görünür güç ifadesi seri gerilim kaynağı tarafından sağlanmakta olup, Denklem (13)'te gösterilmiştir.

$S_{\text {evirici } 2}=v_{s} I_{i j}^{*}=r e^{j \gamma} V_{i}\left(\frac{\bar{V}_{i}^{\prime}-V_{j}}{j X_{s}}\right)^{*}$

Denklem (13)'te yer alan görünür güç ifadesinin sadeleştirilmesiyle elde edilen evirici 2 devresindeki aktif ve reaktif güç ifadeleri Denklem (14) ve Denklem (15)'te gösterilmiştir.

$$
\begin{aligned}
& P_{\text {evirici2 }}=r b_{s} V_{i} V_{j} \sin \left(\theta_{i}-\theta_{j}+\gamma\right)-r b_{s} V_{i}^{2} \sin \gamma \\
& Q_{\text {evirici2 }}=-r b_{s} V_{i} V_{j} \cos \left(\theta_{i}-\theta_{j}+\gamma\right)-r b_{s} V_{i}^{2} \cos \gamma+r^{2} b_{s} V_{i}^{2}
\end{aligned}
$$

Burada, $\theta \mathrm{i}$ ve $\theta \mathrm{j}$ i ve $\mathrm{j}$ numaralı baralardaki gerilim açıları olarak ifade edilir. Reaktif gücün evirici1 tarafından alınması veya verilmesi UPFC tarafından bağımsız olarak kontrol edilebilir. Buna ek olarak, ayrı bir şönt reaktif kaynak olarak modellenebilir. Bu durumda i barasındaki gerilim seviyesini kabul edilebilir sınırlar içerisinde tutmak reaktif gücün temel işlevidir. Sonuç olarak sürekli durum UPFC matematiksel model i ve j baraları arasında bulunan evirici 1 evirici 2 devresi ile yapılandırmaktadır [11]. Yapılandırılan bu model Şekil 3 'te gösterilmiştir. 


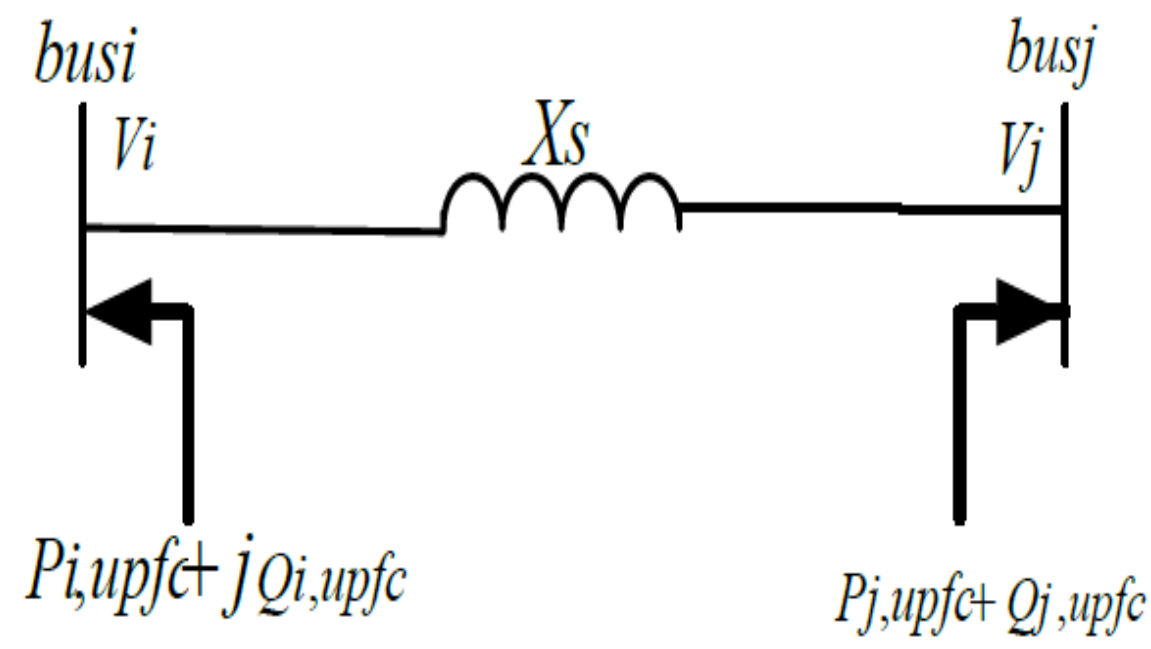

Şekil 4. İki makinall güç sisteminin UPFC ile geliştirilmiş modeli

Şekilde 4'te yer alan eşdeğer güç eşitlikleri Denklem (16), Denklem (17), Denklem (18) ve Denklem (19)' da gösterilmiştir.

$$
\begin{aligned}
& P_{i, \text { uppfc }}=r b_{s} V_{i}^{2} \sin \gamma-r b_{s} V_{i} V_{j} \sin \left(\theta_{i}-\theta_{j}+\gamma\right) \\
& P_{j, \text { uppfc }}=r b_{s} V_{i} V_{j} \sin \left(\theta_{i}-\theta_{j}+\gamma\right) \\
& Q_{i, u p p f c}=r b_{s} V_{i}^{2} \cos \gamma \\
& Q_{j, \text { uppfc }}=r b_{s} V_{i} V_{j} \cos \left(\theta_{i}-\theta_{j}+\gamma\right)
\end{aligned}
$$

\section{GÜC SALINIM SÖNÜMLEMESİ(GSS)}

UPFC geçici durum esnasında güç salınımlarını sönümlemek için kullanılabilir ve böylece geçici durum çalışmadaki sistemin genel dinamik performansını arttırır. Böyle bir işlevi yerine getirirken kullanılan kararlılık kontrol döngüsü elektromekanik geçici durumlar sırasındaki seri olarak enjekte edilen gerilimin büyüklügünü kontrol eden bir üniteden oluşur. Bu şekilde kullanılan cihazlara GSS kontrol cihazı denir. GSS kontrol devresi Şekil 5'de gösterilmiştir.

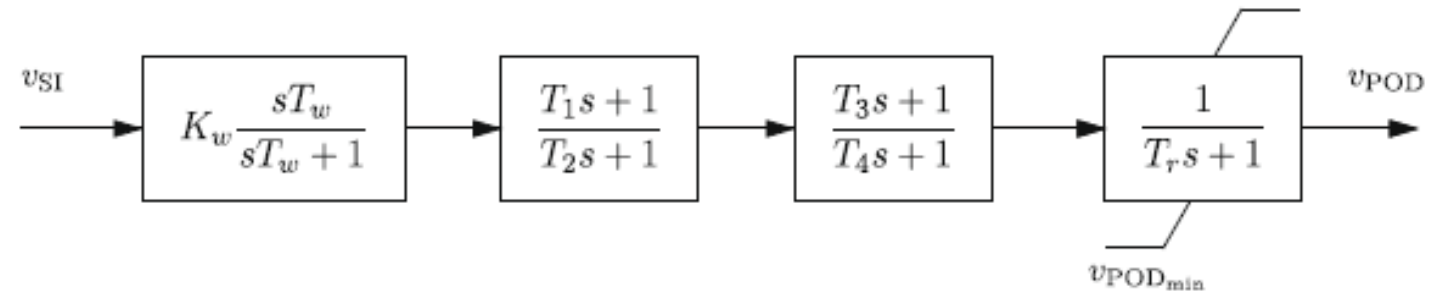

Şekil 5. GSS kontrol devresi

GSS denetleyicisi dengeleyici kazancı, bir arınma filtresi ve faz kompansatör bloklarından oluşur. Arınma sinyali, POD çıkışının sabit durumda sıfır olmasını sağlar. $V_{P O D}$ çıkış sinyali bir anti-wind-up sınırlayıcısına tabi tutulur ve dinamiği küçük bir zaman sabiti tarafından verilir. Kazanç $\mathrm{K}_{\mathrm{w}}$, POD 
tarafindan eklenen sönümleme miktarını belirler ve faz kompansatör blokları, giriş sinyalinin uygun faz gecikme telafisini sağlar [12].

GSS parametrelerinin ayarlanması için birkaç tasarım yöntemi kullanılabilir. En popüler olanları sistemin frekans tepkisi, özdeğer hassasiyeti ve frekans-özdeğer birleşmesine dayanır. Burada GSS kullanılması ile faz ve kazanç marjı tekniklerine dayanan frekans yanıt yöntemini hesaplamaktadır. Temel olarak, tasarım prosedürü iki ana adımdan oluşur. Bunlar telafi edilecek fazın belirlenmesi ve denetleyici kazancının hesaplanmasıdır. Bu çalışma için düşünülen sönümleme oranını bu şekilde elde edilmektedir. Buna bağlı olarak faz kompansatör bloğunun parametrelerinin hesaplanması Denklem (20) ve Denklem (21)'de gösterilmiştir.

$$
\begin{aligned}
& T=\frac{1}{w_{n} \sqrt{\alpha}} \\
& \alpha=\frac{1-\sin (\phi / n)}{1+\sin (\phi / n)}
\end{aligned}
$$

Burada, $\mathrm{T}$ zaman, $\varphi$ kompanse edilecek olan faz, $\mathrm{w}_{\mathrm{n}}$ sönümleme frekansı, $\mathrm{n}$ ise gecikmeli şebeke sayısıdır. Gecikme şebekesi parametrelerinin ayarlandıktan sonra 2. adım gerçekleştirilir. Güç sistemlerinde bozucu etkinin durumuna göre genellikle \% 10 sönüm oranının yeterli olduğu kabul edilir [13].

\section{SENKRON GENERATÖR MODELLERİ}

Senkron generatör modelleri olarak 2. derece modelinde 6. derece modeline kadar olan dinamik siras1 kullanılmaktadır. 2. derece modelinde generatör açısı ve generatör açısal hız ifaelerine yer verilmektedir. Generatör açısı ve generatör açısal hız hesaplamaları denklem (22) ve denklem (23)'de gösterilmiştir.

$$
\begin{aligned}
& \delta=f_{b}(\omega-1) \\
& \omega=\left(P_{m}-P_{e}-D(\omega-1)\right) / M
\end{aligned}
$$

Alt-geçici durum dinamik derece modelinde açı, açısal hız ve tüm q ekseni elektromanyetik devreleri ihmal edilmesi ile ve d ekseni indüktansı ilave edilmiştir. Dahası tüm yukarıda ifade edilenlerin yanısıra, $\mathrm{d}$ eksenine bir devre ve q eksenine iki devrenin ilave edilmesi ile elde edilir. Alt-geçici durum dinamik derece modelleri altı değişkenden oluşur. Bunlar; açı, açısal hız, q eksen geçici gerilim kaynağı, d eksen geçici gerilim kaynağı, q eksen alt geçici gerilim kaynağı, d eksen alt geçici gerilim kaynağıdır. Senkron generatörlerde alt-geçici durum dinamik derece modelinin kullanılması ile elde edilmesinde kullanılan ifadeler Denklem (24) ile Denklem (29) arasında gösterilmiştir.

$$
\begin{aligned}
& Q_{s}=\frac{V_{s}^{2}}{X_{t}}+\frac{V_{s} V_{t}}{X_{t}} \sqrt{1-\left(\frac{X_{t} I_{k}}{K_{m} V_{s}}\right)^{2}} \\
& e_{g 0}=-\frac{X_{t}}{V_{s}\left(V_{t} / K_{m}\right)} \sqrt{P_{g}^{2}+\left(Q_{q}+\frac{V_{g}^{2}}{X_{t}}\right)^{2}} \\
& e_{q}^{\prime}=\left[-e_{q}^{\prime}-\left(x_{d}-x_{d}^{\prime}-\frac{T_{d 0}^{\prime \prime} x_{d}^{\prime \prime}}{T_{d 0}^{\prime} x_{d}^{\prime}}\left(x_{d}-x_{d}^{\prime}\right)\right) i_{d}+\left(1+\frac{T_{A A}}{T_{d 0}^{\prime \prime}}\right) v_{f}^{*}\right] / T_{d 0}^{\prime}
\end{aligned}
$$




$$
\begin{aligned}
& e_{d}^{\prime}=\left[-f_{s}\left(e_{d}^{\prime}\right)+\left(x_{q}-x_{q}^{\prime}-\frac{T_{q 0}^{\prime \prime} x_{q}^{\prime \prime}}{T_{q 0}^{\prime} x_{q}^{\prime}}\left(x_{q}-x_{q}^{\prime}\right)\right) i_{q}\right] / T_{q 0}^{\prime} \\
& e_{q}^{\prime \prime}=\left[-e_{q}^{\prime \prime}+e_{q}^{\prime}-\left(x_{d}^{\prime}-x_{d}^{\prime \prime}-\frac{T_{d 0}^{\prime \prime} x_{d}^{\prime \prime}}{T_{d 0}^{\prime} x_{d}^{\prime}}\left(x_{d}-x_{d}^{\prime}\right)\right) i_{d}+\left(\frac{T_{A A}}{T_{d 0}^{\prime \prime}}\right) v_{f}^{*}\right] / T_{d 0}^{\prime \prime} \\
& e_{d}^{\prime \prime}=\left[-e_{d}^{\prime \prime}+e_{d}^{\prime}+\left(x_{q}^{\prime}-x_{q}^{\prime \prime}-\frac{T_{q 0}^{\prime \prime} x_{q}^{\prime \prime}}{T_{q 0}^{\prime} x_{q}^{\prime}}\left(x_{q}-x_{q}^{\prime}\right)\right) i_{q}\right] / T_{q 0}^{\prime}
\end{aligned}
$$

Burada, $\mathrm{f}_{\mathrm{b}}$ temel frekans, $\mathrm{P}_{\mathrm{m}}$ mekanik güç, $\mathrm{M}$ moment, $\mathrm{D}$ sönümleme katsayıs1, $\mathrm{x}_{\mathrm{d}}$ and $\mathrm{x}_{\mathrm{q}} \mathrm{d}$-q eksen senkron reaktanslar, $x_{d}^{\prime}$ and $x^{\prime}{ }_{q} d-q$ eksen senkron geçici reaktanslar, $x^{\prime \prime}$ and $x^{\prime \prime} d-q$ eksen senkron alt geçici reaktanslar, $\mathrm{T}_{\mathrm{d} 0}$ and $\mathrm{T}_{\mathrm{q} 0} \mathrm{~d}$-q eksen açık devre geçici zaman sabiti, $\mathrm{T}_{\mathrm{d} 0}{ }$ and $\mathrm{T}{ }_{\mathrm{q} 0} \mathrm{~d}-\mathrm{q}$ eksen açık devre alt geçici zaman sabiti, $\mathrm{T}_{\mathrm{AA}} \mathrm{d}-\mathrm{q}$ eksen ilave kaçak zaman sabiti, $\mathrm{i}_{\mathrm{d}}$ and $\mathrm{i}_{\mathrm{q}} \mathrm{d}$-q eksen akımı, $\delta$ rotor açısı, w rotor hızı, $v_{f}$ alan gerilimi, $e_{d}^{\prime}$ and $e_{q}^{\prime} d-q$ eksen geçici gerilim kaynağ $1, e^{\prime \prime}{ }_{d}$ and e ${ }_{q} d-q$ eksen alt geçici gerilim kaynağıdır[14].

\section{BENZETIM CALISMASI}

Bu çalışmada kundur 4 makinalı, 2 alan test sistemi kullanılmıştır. Kundur 4 makinalı, 2 alan test sistemi Şekil 6'da gösterilmiştir.

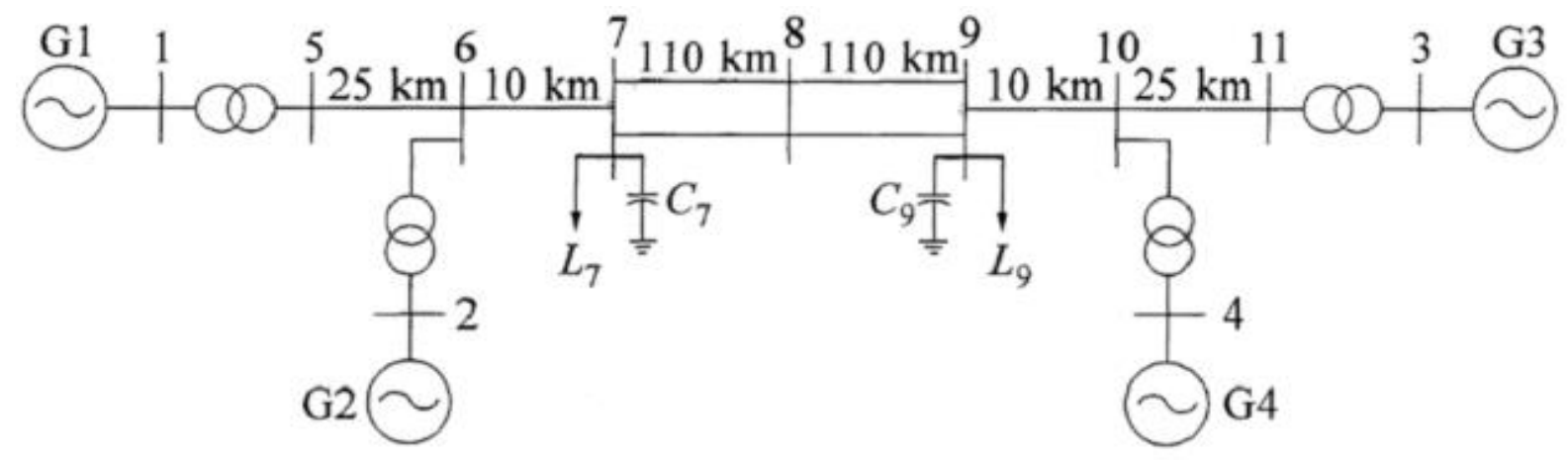

Şekil 6. Kundur 4 makinall, 2 alan test sistemi

$\mathrm{Bu}$ test sisteminde 4 senkron generatör kullanılmıştır. G1 ve G2 generatörlerinin olduğu kısım 1. alan olurken, G3 ve G4 generatörlerinin olduğu kısım 2. alanı oluşturmaktadır. Sistemde toplamda 11 tane bara kullanılmıştır. 1. bara hem salınım barası hemde generatör barası olarak kullanılırken, 2, 3, 4 numaralı baralar generatör barası, geriye kalan diğer 7 bara yük barası olarak kullanılmaktadır. 7 ve 9 numaralı baralarda statik kondenser kullanılmıştır. Ayrıca 7 ve 9 numaralı baralarda statik yük olarak kullanılan ZIP yük modelleri bağlanmıştır. Bu sistemde ayrıca 4 adet transformatör kullanılmaktadır. 5 ile 6 numaralı baralar arası mesafe $25 \mathrm{~km}, 6$ ile 7 numaralı baralar arası mesafe $10 \mathrm{~km}, 7$ ile 8 numaralı baralar arası mesafe $110 \mathrm{~km}, 8$ ile 9 numaralı baralar arası mesafe $110 \mathrm{~km}, 9$ ile 10 numaralı baralar aras1 $25 \mathrm{~km}, 10$ ile 11 numaralı baralar arası mesafe $25 \mathrm{~km}$ olarak belirlenmiştir. Bu sistem için kullanılan UPFC'nin gücü 100 MVA olarak seçilmiştir. Bu sistemde 3 faz arızası eydana gelmiştir. 3 faz arızası 8 numaralı barada gerçekleşmiştir. 8 numaralı bara ile 9 numaralı bara arasında kesici kullanılmıştır. Arıza süresi 1 saniye ile 1.05 saniyeler arasındadır. 100 MVA gücündeki UPFC 8 ile 9 numaralı baralar arasına bağlanmıştır. Kesici açma kapama süresi 1.05-1.1 saniyeler arasında belirlenmiştir. Ayrıca senkron generatörlerde otomatik gerilim regülatörü ve türbin yöneticisi modelleri 
de kullanılmıştır. GSSC modeli 7 ile 8 numaralı baralar arasında bulunmaktadır. GSSC modelde kullanılan parametre değerleri Tablo 1'de gösterilmiştir.

Tablo 1. GSSC modelde kullanilan parametre değerleri

\begin{tabular}{cc}
\hline Sembol & Değeri \\
\hline $\mathrm{V}_{\min }$ & -0.12 p.u. \\
\hline $\mathrm{V}_{\max }$ & 0.12 p.u. \\
\hline $\mathrm{K}_{\mathrm{w}}$ & -0.577 \\
\hline $\mathrm{T}$ & 10 \\
\hline $\mathrm{T}_{1}$ ve $\mathrm{T}_{2}$ & 0.3187 ve 0.1928 \\
\hline
\end{tabular}

Senkron generatörlerin alt-geçici durum dinamik derece modellinin diğer bir adı 6.derece modelidir. Benzetim çalışması 2 analiz yönteminde oluşmaktadır. İlk analiz UPFC, GSSC'nin olmadığı durum ve senkron generatörlerin 2.derece modelde kullanımıdır. İkinci analizde ise UPFC, GSSC'nin olduğu durum ve senkron generatörlerin 6.derece modelde kullanımıdır.

\section{BENZETIM CALISMASI SONUCLARI}

İlk yapılan analizde UPFC, GSSC'nin olmadığ 1 durum ve senkron generatörlerin 2.derece modeldeki analizidir. İlk analiz sonucunda senkron generatör açısal hız değişimleri, senkron generatör aktif-reaktif güç değişimleri, generatör barası gerilim değişimleri Şekil 7 ile Şekil 10 arasında gösterilmiştir.

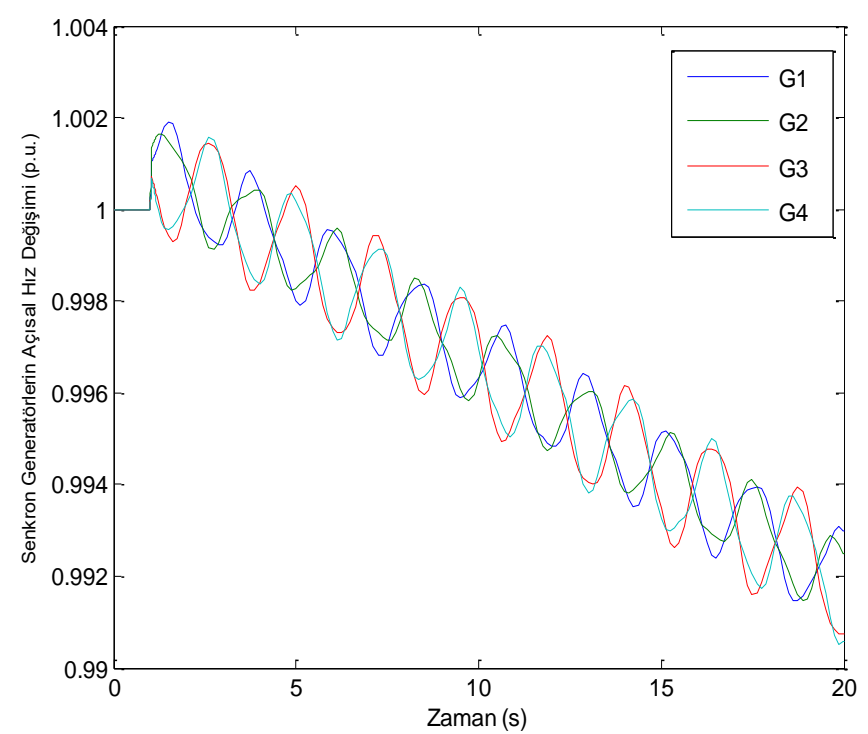

Şekil 7. UPFC, GSSC'nin olmadiğı durum ve senkron generatörlerin 2.derece modeldeki açısal hız değişimleri 


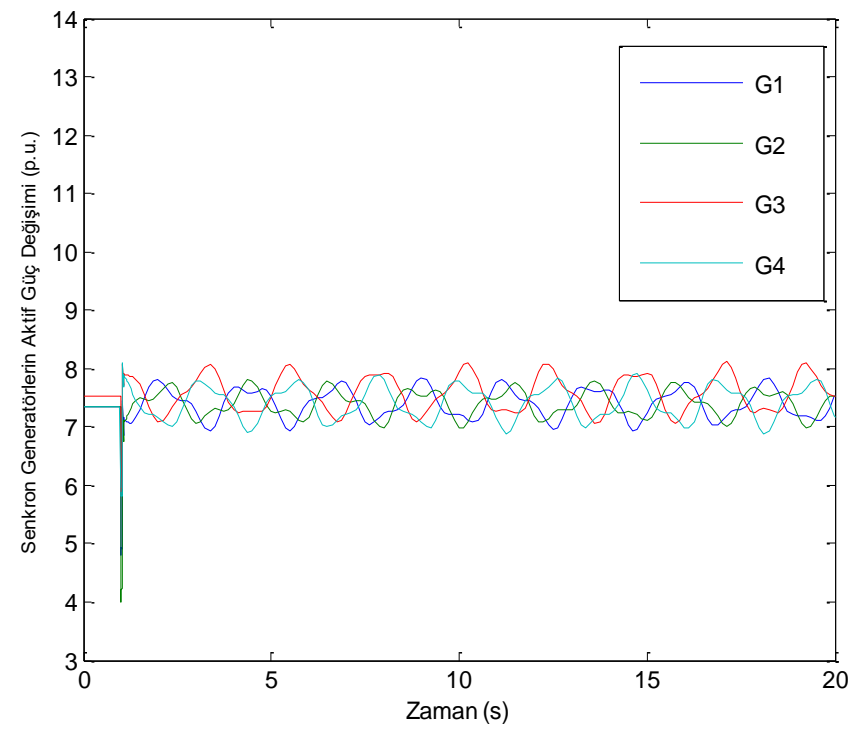

Şekil 8. UPFC, GSSC'nin olmadı̆ğ durum ve senkron generatörlerin 2.derece modeldeki aktif gü̧̈ değişimleri

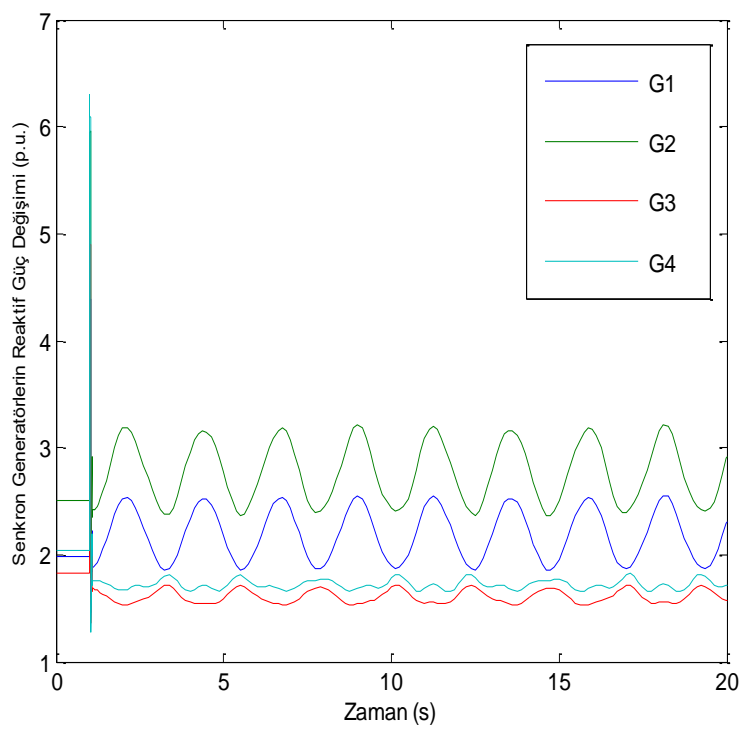

Şekil 9. UPFC, GSSC'nin olmadĭ̆ durum ve senkron generatörlerin 2.derece modeldeki reaktif güç değişimleri 


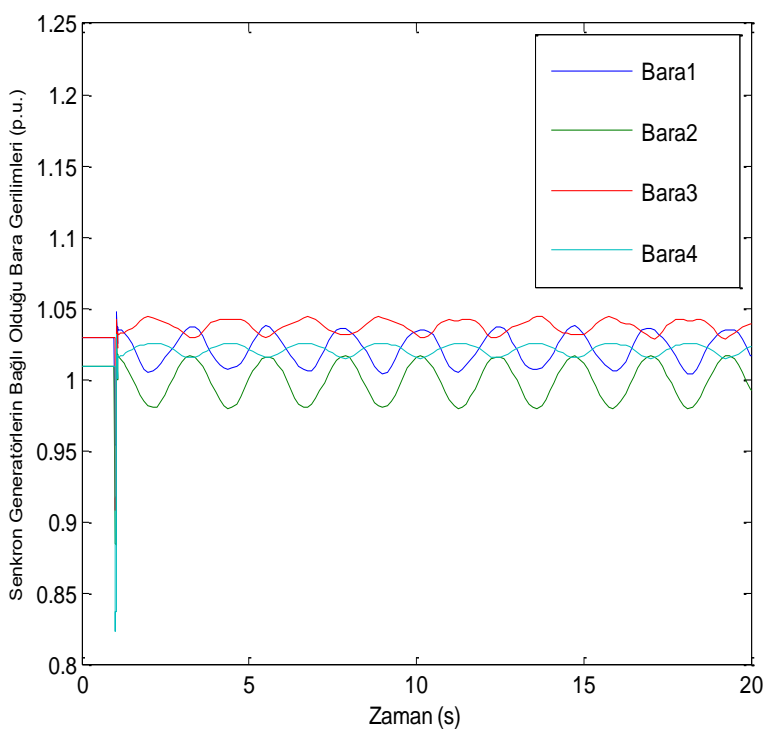

Şekil 10. UPFC, GSSC'nin olmadı̆g durum ve senkron generatörlerin 2.derece modeldeki generatör barası gerilim değişimleri

Yapılan ilk analiz sonucunda senkron generatör açısal hızının, aktif-reaktif güç değişimlerinin ve generatör baralarının gerilim değişlimlerinde arıza sonrasında kararsızlığa gitti görülmektedir. $\mathrm{Bu}$ parametreler içerisinde salınım açısından en çok etkilenen parametre senkron generatör aktif güç değişimi olurken, en az etkilenen parametre senkron generatörlerin bağlı olduğu bara gerilim değişimleridir. İkinci yapılan analizde UPFC, GSSC'nin olduğu durum ve senkron generatörlerin 6.derece modeldeki analizidir. İkinci analiz sonucunda elde edilen generatör açısal hız değişimleri, senkron generatör aktif-reaktif güç değişimleri, generatör barası gerilim değişimleri Şekil 11 ile Şekil 14 arasında gösterilmiştir.

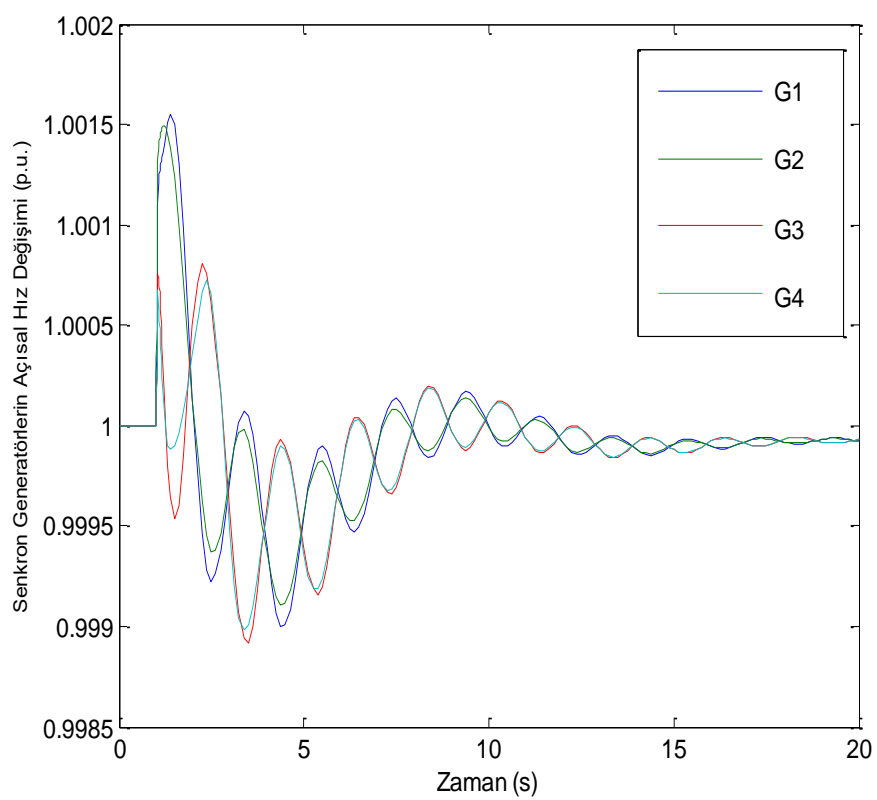

Şekil 11. UPFC, GSSC’nin olduğu durum ve senkron generatörlerin 6.derece modeldeki açısal hız değişimleri 


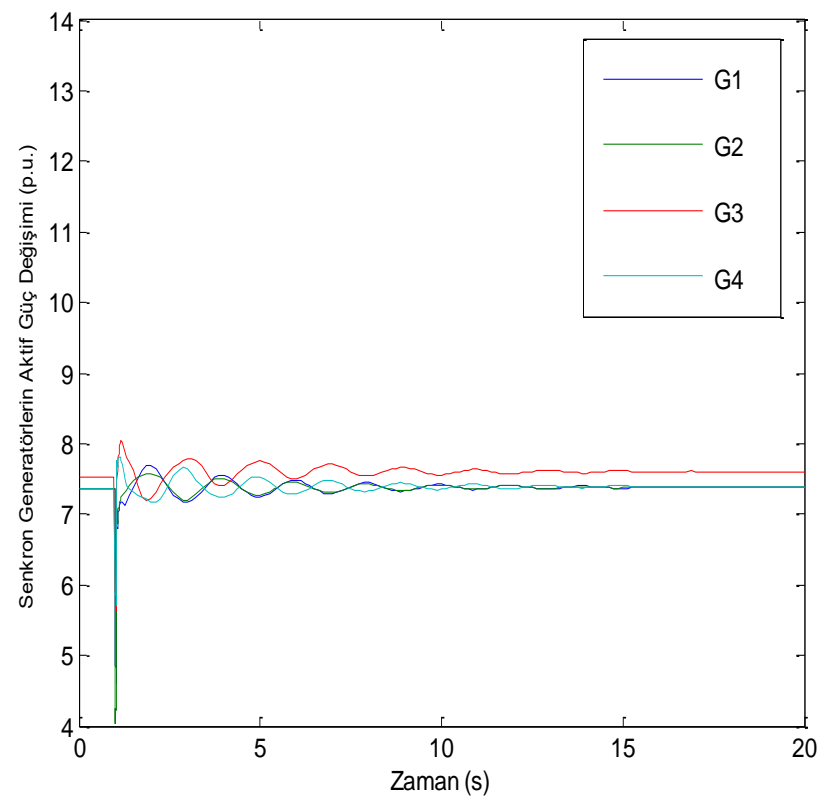

Şekil 12. UPFC, GSSC'nin olduğu durum ve senkron generatörlerin 6.derece modeldeki aktif gü̧̧ değişimleri

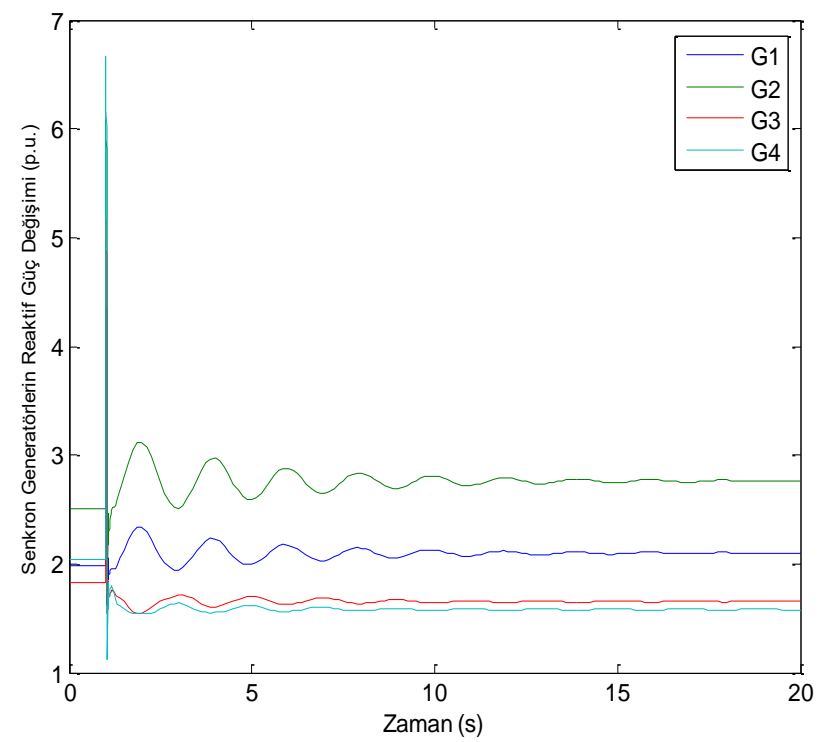

Şekil 13. UPFC, GSSC'nin olduğu durum ve senkron generatörlerin 6.derece modeldeki reaktif gü̧̈ değişimleri 


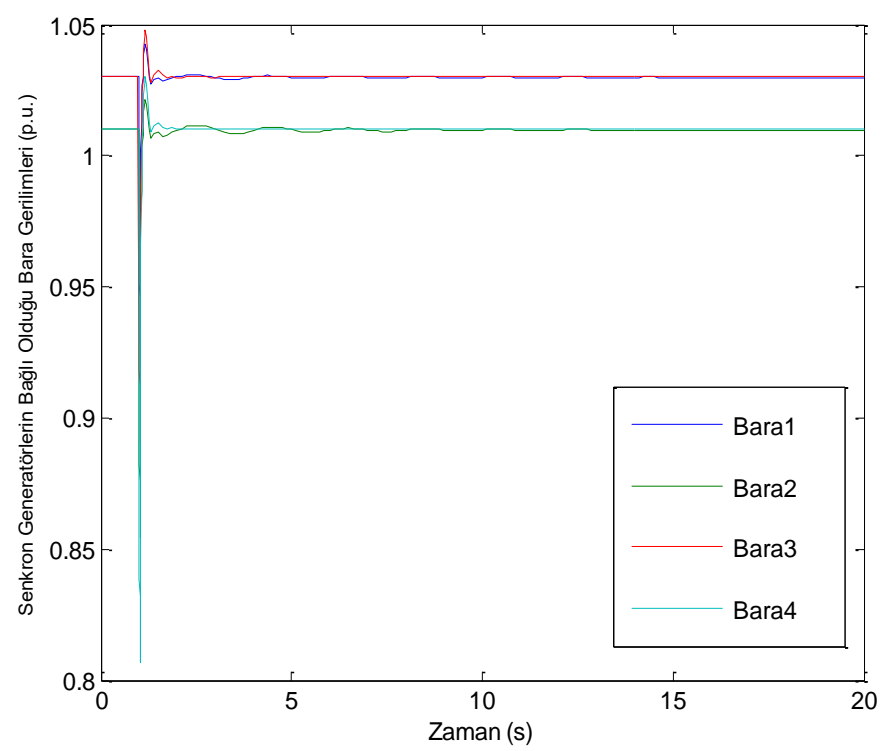

Şekil 14. UPFC, GSSC'nin olduğu durum ve senkron generatörlerin 6.derece modeldeki generatör barası gerilim değişimleri

Yapılan ikinci analiz sonucunda senkron generatör açısal hızının, aktif-reaktif güç değişimlerinin ve generatör baralarının gerilim değişimlerinde arıza sonrasında sistemin kararlı hale geldiği görülmüştür. $\mathrm{Bu}$ parametreler içerisinde salınım açısından en çok etkilenen parametre senkron generatör aktif güç değişimi olurken, en az etkilenen parametre senkron generatörlerin bağlı olduğu bara gerilim değişimleridir.

\section{SONUCLAR}

$\mathrm{Bu}$ çalışmada FACTS cihazlarının içerisinde en gelişmiş model UPFC ile GSSC kontrolü ve senkron generatörün alt-geçici derece modelinin birlikte kullanılmasın ile 3 faz arıza analizindeki etkileri incelenmiştir. Kundur 4 makinalı 2 alanlı sistemde yapılan analizlerde bu çalışma için geliştirilen modellerin etkili sonuçlar verdiği görülmüştür. Sistemde incelenen senkron generatör açısal hız, senkron generatörlerin aktif-reaktif güçleri ve senkron generatörlerin bağlı olduğu bara gerilim değişimleri UPFC, GSSC ve alt geçici derece modelleri sayesinde kısa sürede kararlı hale gelirken, salınımların kısa süre içerisinde sönümlendiği görülmüştür. Yapılan bu çalışma ile diğer FACTS cihazlarının farklı test sistemleri için GSSC'nin kullanımına ve senkron generatör kontrolünde kullanılan çeşitli modellerin geliştirilmesine zemin hazırlamış bulunmaktadır.

\section{KAYNAKLAR}

[1] R. K. Pandey ve N. K. Singh, "UPFC control parameter identification for effective power oscillation damping," International Journal of Electrical Power \& Energy Systems, c. 31, s. 6, ss. 269276, 2009.

[2] M. A. Furini, A. L. S. Pereira, ve P. B. Araujo, "Pole placement by coordinated tuning of power system stabilizers and FACTS-POD stabilizers," International Journal of Electrical Power \& Energy Systems, c. 33, s. 3, ss. 615-622, 2011. 
[3] S. N. Dhurvey, ve V. K. Chandrakar, "Performance comparison of UPFC in co-ordination with optimized POD and PSS on damping of power system oscillations," WSEAS Trans on Power System, c. 3, s. 5, ss. 287-299, 2008.

[4] M. Sobha, R. S. Kumar, ve S. George, "ANFIS based coordination of multiple UPFC control functions for damping low frequency oscillations in power systems," International Energy Journal, c. 9, s. 4, ss. 291-302, 2009.

[5] N. Anwer, A. S. Siddiqui, ve A. Umar, "Analysis of UPFC, SSSC with and without POD in congestion management of transmission system," In 2012 IEEE 5th India International Conference on Power Electronics (IICPE), 2012, ss. 1-6.

[6] S. K. Samal, ve P. C. Panda, "Damping of power system oscillations by using unified power flow controller with POD and PID controllers," In 2014 International Conference on Circuits, Power and Computing Technologies [ICCPCT-2014], 2014, ss. 662-667.

[7] P. Krishnagandhi, "Power oscillation damping of two machine system," In 2014 IEEE 8th International Conference on Intelligent Systems and Control (ISCO), 2014, ss. 167-171.

[8] L. F. B. Martins, P. B. de Araujo, E. de Vargas Fortes, ve L. H. Macedo, "Design of the PIUPFC-POD and PSS damping controllers using an artificial bee colony algorithm," Journal of Control, Automation and Electrical Systems, c. 28, s. 6, ss.762-773, 2017.

[9] C. R. Makkar, ve L. Dewan, "Simultaneous coordination of power system stabilizer and UPFC for improving dynamic stability of multimachine system," In 2014 IEEE 6th India International Conference on Power Electronics (IICPE), 2014, ss. 1-4.

[10] B. E. Maleki, ve H. Beiranvand, "A novel method of FACTS-POD design to more enhancement of inter-area mode damping in a multi-machine power system," Journal of Electrical and Computer Engineering Innovations, c. 6, s. 1, ss. 95-106, 2018.

[11] M. Sarkar, "Load flow studies with UPFC power injection model," Master Thesis, Department of Electrical Engineering National Institute of Technology Rourkela, Hindistan, 2013.

[12] H. M. Ayres, I. Kopcak, M. S. Castro, F. Milano, ve V. F. Da Costa, "A didactic procedure for designing power oscillation dampers of FACTS devices," Simulation Modelling Practice and Theory, c. 18, s. 6, ss. 896-909, 2010.

[13] M. S. Castro, H. M. Ayres, V. F. Da Costa, ve L. C. P. Da Silva, "Impacts of the SSSC control modes on small-signal and transient stability of a power system," Electric Power Systems Research, c.77, s. 1, ss. 1-9, 2007.

[14] F. Milano, "An open source power system analysis toolbox," IEEE Transactions on Power Systems, c. 20, s. 3, ss. 1199-1206, 2005. 